01

\title{
Потенциал ионизации металлического кластера, содержащего вакансии
}

\author{
(C) В.В. Погосов, В.И. Рева \\ Запорожский национальный технический университет, \\ Запорожье, Украина \\ E-mail: vpogosov@zntu.edu.ua
}

(Поступила в Редакцию 5 августа 2016 г.)

Предлагается последовательная процедура нахождения потенциала ионизации большого металлического кластера радиуса $R_{N, v}$, состоящего из $N$ атомов и содержащего $N_{v}$ вакансий. В приближении эффективной среды для энергии основного состояния электронов построена теория возмущения по малым параметрам $R_{v} / R_{N, v}$ и $L_{v} / R_{v}\left(R_{v}\right.$ и $L_{v}$ - среднее расстояние между вакансиями и длина рассеяния электрона на вакансии и соответственно). Профиль эффективного потенциала вакансии, фазы и длина рассеяния электрона предварительно вычислены методом Кона-Шема для макроскопического металла в модели стабильного желе. Полученные аналитические зависимости могут быть полезными при проведении анализа результатов фотоионизационных экспериментов и для определения размерной зависимости концентрации вакансий, в том числе вблизи температуры плавления.

DOI: 10.21883/FTT.2017.06.44474.383

\section{1. Введение}

Фазовый переход из твердого в жидкое состояние в 3D-образцах связан с конфигурационным возбуждением - образованием пустот-вакансий [1,2]. Равновесная концентрация вакансий вычисляется из термодинамических соображений при наличии энергии образования вакансии. Эта величина может быть извлечена из спектра аннигиляции позитронов, локализованных в вакансии. Концентрация вакансий при этом остается свободным параметром [3].

Экспериментально установлено, что температура плавления свободных кластеров уменьшается с уменьшением их размеров. В процессе плавления диффузия поверхностных вакансий в объем более выгодна для кластеров с незаполненными электронными оболочками, чем для магических кластеров [4]. Существует точка зрения, что вблизи температуры плавления энергия образования вакансий тем меньше, чем меньше кластер, а концентрация вакансий не зависит от его размеpa $[5,6]$. Тем не менее, несмотря на детальные массспектрометрические и калориметрические исследования процесса предплавления и плавления кластеров (см., например, [4,7,8]), вопрос о размерной зависимости энергии образования вакансий, их концентрации и связи с процессом плавления остается открытым.

Впервые масс-спектрометрические измерения энергии диссоциации кластерных ионов $\mathrm{Na}_{N}^{+}$проведены в [9], а для $\mathrm{Al}_{N}^{+}-$в [10]. Традиционно по таким данным, а также по измеренным потенциалам ионизации рассчитывается энергия когезии нейтральных кластеров. Поэтому одной из актуальных задач, которые можно сформулировать в связи с плавлением малоразмерных агрегатов, является определение их потенциала ионизации.

Недавно потенциал ионизации $\mathrm{Al}_{N} \quad(N=32-95)$ и его температурная зависимость в диапазоне тем- ператур 65-230 K измерялись в [11]. С ростом температуры отмечается в среднем незначительное ( $\sim 10 \mathrm{meV})$ уменьшение потенциала ионизации. Температура плавления таких кластеров находится в интервале $600-700 \mathrm{~K}[7,8]$.

Для кластеров со счетным числом атомов энергия связи определяется численными методами. Для больших кластеров энергия связи обычно определяется по энергии основного состояния в протяженном конденсированном материале с учетом первой размерной поправки $\sim R_{N}^{-1}$, обусловленной кривизной поверхности $[12,13]$.

В настоящей работе впервые учитываются поправки $\sim R_{N}^{-2}$. Помимо этого разработана процедура, пригодная для вычисления потенциала ионизации и энергии прилипания электронов металлических кластеров, содержащих точечные дефекты и примеси. В качестве примера выбраны вакансии. Данный подход может быть легко адаптирован для расчета энергии связи позитрона в таких кластерах.

Методически задача решалась в два этапа. На первом этапе для полубесконечного 3D-металла с плоской поверхностью (радиус кривизны равен нулю) методом Кона-Шема рассчитываются все его характеристики, включая энергию образования объемной вакансии. В модели стабильного желе моновакансию представляют в виде сферической полости атомного размера в однородном положительно заряженном фоне, создаваемом ионами. Эта задача выполнена в работах $[14,15]$. В результате был найден вклад разреженной системы вакансий в работу выхода электронов [14] и позитронов $[3,15]$ в 3D-металле. На втором этапе эти данные используются нами для построения асимптотической размерной зависимости потенциала ионизации сферического металлического кластера энергии прилипания электрона. 


\section{2. Основные соотношения}

Представим кластер в виде сферы. Потенциал ионизации и энергия прилипания электронов для металлического кластера с числом атомов $N$, валентностью $Z$ и радиусом

$$
R_{N}=N^{1 / 3} r_{0}
$$

по определению имеют вид

$$
\begin{gathered}
\mathrm{IP}_{N}=E_{N}^{N_{e}-1}\left(R_{N}\right)-E_{N}^{N_{e}}\left(R_{N}\right), \\
\mathrm{EA}_{N}=E_{N}^{N_{e}}\left(R_{N}\right)-E_{N}^{N_{e}+1}\left(R_{N}\right),
\end{gathered}
$$

где $E_{N}^{N_{e}-1}\left(E_{N}^{N_{e}+1}\right)$ - полная энергия сферы с избыточным зарядом $+e(-e)$. Здесь $N_{e}=Z N-$ полное число валентных электронов в нейтральном кластере.

Основой данного подхода является разложение химического потенциала $\mu$ валентных электронов нейтрального кластера по степеням обратного радиуса $R_{N}^{-1}$ и соответствующее разложение удельной поверхностной энергии $\sigma$ (liquid drop model)

$$
\begin{aligned}
& \mu\left(R_{N}\right)=\mu_{0}+\frac{\mu_{1}}{R_{N}}+\frac{\mu_{2}}{R_{N}^{2}}+O\left(R_{N}^{-3}\right), \\
& \sigma\left(R_{N}\right)=\sigma_{0}+\frac{\sigma_{1}}{R_{N}}+\frac{\sigma_{2}}{R_{N}^{2}}+O\left(R_{N}^{-3}\right),
\end{aligned}
$$

где величины $\mu_{0}$ и $\sigma_{0}$ отвечают плоской поверхности $\left(R_{N} \rightarrow \infty\right)$.

Из условия механического равновесия кластера в $[16,17]$ получены правила сумм, в частности

$$
\begin{gathered}
\mu_{1}=\frac{2 \sigma_{0}}{\bar{n}}, \\
\mu_{2}=\frac{2 \sigma_{1}}{\bar{n}}-\frac{\mu_{1}^{2} \bar{n}}{2 B_{0}} \equiv \mu_{1}\left(\delta_{1}-\frac{\sigma_{0}}{B_{0}}\right),
\end{gathered}
$$

где $\delta_{1}=\sigma_{1} / \sigma_{0}, B_{0}$ - объемный модуль сжатия 3Dметалла, $\bar{n}=3 Z /\left(4 \pi r_{0}^{3}\right)$ - концентрация электронов в металле, $r_{0}$ - радиус ячейки Вигнера-Зейтца, приходящейся на один атом.

Если в кластере содержится $N_{v}$ вакансий, тогда его радиус

$$
R_{N, v}=\left(N+N_{v}\right)^{1 / 3} r_{0} \approx R_{N}\left(1+\frac{1}{3} c_{v}\right),
$$

относительная концентрация вакансий $c_{v}=N_{v} / N=$ $=\left(r_{0} / R_{v}\right)^{3}$, где $R_{v}$ - радиус сверхъячейки ВигнераЗейтца, приходящейся на одну вакансию в кластере. Предполагается, что $R_{N, v} \gg R_{v}$.

По аналогии для кластера с вакансиями в асимптотическом приближении используем выражения

$$
\begin{gathered}
\mathrm{IP}_{N, v}=W_{v}^{3 \mathrm{D}}-\frac{\mu_{1}}{R_{N, v}}-\frac{\mu_{2}}{R_{N, v}^{2}}+\frac{e^{2}}{2 C_{N, v}}, \\
\mathrm{EA}_{N, v}=W_{v}^{3 \mathrm{D}}-\frac{\mu_{1}}{R_{N, v}}-\frac{\mu_{2}}{R_{N, v}^{2}}-\frac{e^{2}}{2 C_{N, v}}, \\
W_{v}^{3 \mathrm{D}}=W_{0}+\delta W_{v},
\end{gathered}
$$

где $W_{0}=-\mu_{0}-$ работа выхода электронов из бездефектного 3D-металла, состоящая из объемной компо- ненты и поверхностного дипольного барьера. Величина $\delta W_{v}$ - поправка к объемной компоненте работы выхода, обусловленная наличием вакансий $[14,15]$ и пропорциональная их относительной концентрации $c_{v}$. При условии $\left|\delta W_{v}\right| \ll W_{0}$ предполагается слабая зависимость дипольного барьера на внешней поверхности образца от наличия вакансий в его объеме. Поэтому дальнейшая задача сводится к определению сдвига энергии основного состояния $\varepsilon_{b}$ электрона, обусловленного вакансиями нейтрального кластера,

$$
\varepsilon_{b}=-\delta W_{v} .
$$

Последнее слагаемое для IP в (6) - энергия однократно заряженной сферы с электрической емкостью

$$
C_{N, v}=R_{N, v} \approx C_{N}\left(1+\frac{1}{3} c_{v}\right)
$$

в соответствии с определением (5).

\section{3. Теория возмущений в ячеечном приближении}

Разобьем условно кластер на $N_{v}$ сферических сверхъячеек Вигнера-Зейтца радиуса $R_{v}$ по числу вакансий, $N_{v} R_{v}^{3}=R_{N, v}^{3}$. Сверхъячеечный muffin-tin потенциал заменяется на сферически симметричный.

Волновая функция основного состояния электрона в кластере, состоящем из $N$ атомов и $N_{v}$ вакансий, определяется волновым уравнением

$$
\begin{aligned}
-\frac{\hbar^{2}}{2 m} \nabla^{2} \Psi(\mathbf{r}) & +\left[v_{\mathrm{eff}}(r)+\sum_{i=1}^{N_{v}} \delta v_{\mathrm{eff}, v}\left(\mathbf{r}-\mathbf{R}_{i}\right)\right] \Psi(\mathbf{r}) \\
& =\varepsilon_{b} \Psi(\mathbf{r})
\end{aligned}
$$

в котором вакансии центрированы в точках $\mathbf{R}_{i}$, $R_{i}<R_{N, v}$. Одноэлектронный эффективный потенциал (предварительно вычисленный в модели стабильного желе) намеренно разделен таким образом, что сферически симметричный потенциал $v_{\text {eff }}(r)$ формирует дно зоны проводимости и поверхностный барьер, а $\delta v_{\mathrm{eff}, v}\left(\mathbf{r}-\mathbf{R}_{i}\right)-i$-ю вакансию (рис. 1). Энергетическая щель $\varepsilon_{b}$ обусловлена сдвигом энергии основного состояния вследствие наличия вакансий.

Предположим, что потенциальное поле обладает „сферической периодичностью“. Тогда, как это принято в теории конденсированного состояния, удобно представить волновую функцию электрона в основном состоянии $\Psi(\mathbf{r})$ в виде произведения двух функций

$$
\Psi(\mathbf{r})=\psi_{c}(\mathbf{r}) u(\mathbf{r}) .
$$

Функция $\psi_{c}(\mathbf{r})$, меняющаяся на масштабе всего кластера, модулируется на межвакансионных расстояниях функцией $u(\mathbf{r})$. Функция $u(\mathbf{r})$ внутри сверхъячейки, центрированной, например, в точке $\mathbf{R}_{i}$, будет совпадать 


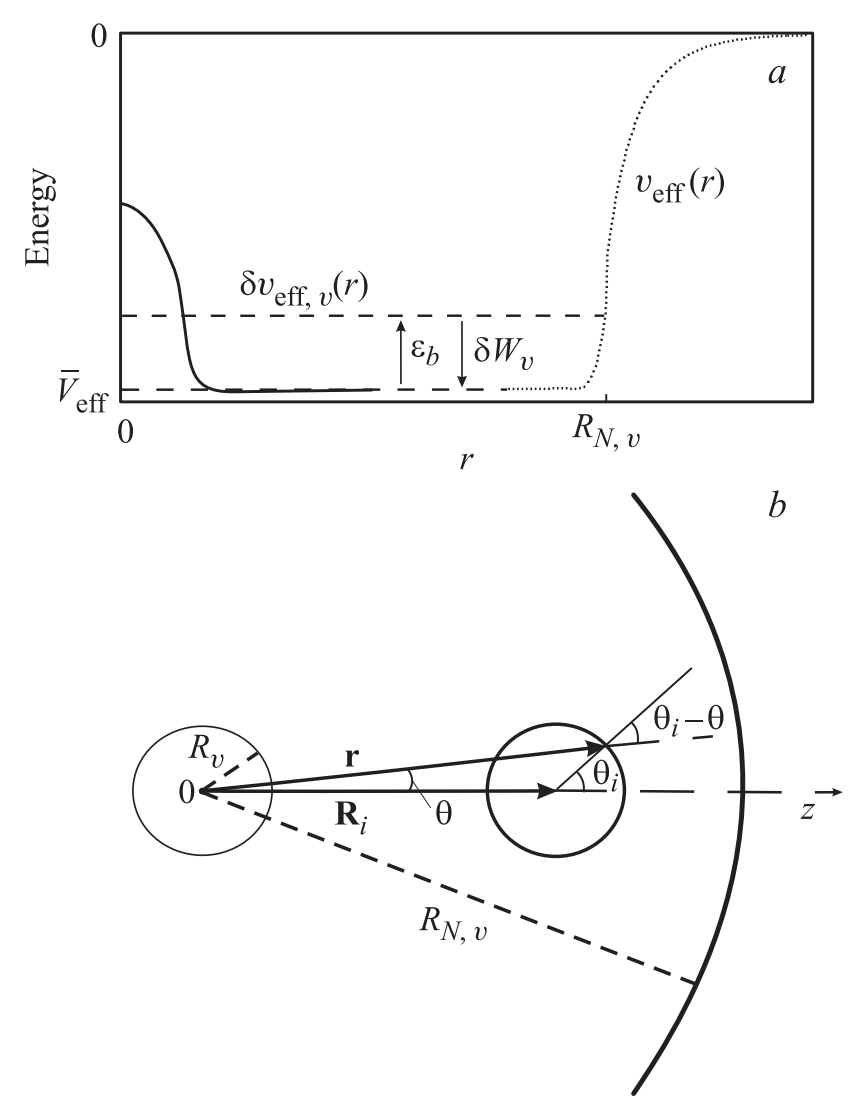

Рис. 1. Энергетическая диаграмма кластера с вакансией в центре $(a)$ и схема для вычисления поверхностного интеграла $i$-й сверхъячейки в теории возмущений $(b)$.

с волновой функцией $u_{\mathrm{WS}}\left(\left|\mathbf{r}-\mathbf{R}_{i}\right|\right)$, удовлетворяющей уравнению Шредингера в сверхъячейке Вигнера-Зейтца:

$$
u(\mathbf{r})=u_{\mathrm{WS}}, \quad r \leq R_{v} .
$$

Далее величину $\varepsilon_{b}$ представим в виде суммы

$$
\varepsilon_{b}=\varepsilon^{(0)}+\varepsilon^{(1)},
$$

где

$$
\varepsilon^{(0)}=T_{0}+\left\langle\delta v_{\text {eff, } v}\right\rangle_{R_{v}},
$$

где $T_{0}$ - энергия основного состояния электрона вследствие вытесненного объема вакансии, вычисляемая в приближении потенциала нулевого радиуса; $\left\langle\delta v_{\text {eff, } v}\right\rangle_{R_{v}}-$ усредненный по объему кластера вклад потенциальной энергии от электрон-вакансионного потенциала $\delta v_{\text {eff, } v}(r) \equiv v_{\text {eff, } v}(r)-\bar{v}_{\text {eff }}$, где $v_{\text {eff }, v}(r)-$ самосогласованный одноэлектронный профиль потенциала, вычисленный методом Кона-Шема в присутствии вакансии, $\bar{v}_{\text {eff }}$ - положение дна зоны проводимости в отсутствие вакансии (рис. $1, a)$.

Мерой радиуса действия потенциала нулевого радиуса служит длина рассеяния электронов на вакансиях $L_{v}$ (она же является и радиусом псевдопотенциала для $s$-фазы рассеяния) на вакансионном потенциале.
Для электрона вакансия представляет собой потенциальный бугор, поэтому $L_{v}>0$ (в боровских радиусах $L_{v}=1.85 a_{0}$ и $1.93 a_{0}$ для $\mathrm{Na}$ и $\mathrm{Al}$ соответственно). Если $R_{N, v} \gg L_{v}$, взаимодействие электрона с вакансиями может быть описано с помощью оптического приближения Ферми

$$
T_{0}=\frac{3 \hbar^{2} L_{v}}{2 m r_{0}^{3}} c_{v} .
$$

Среднее поле сверхъячеек. Энергия основного состояния электрона, например, в центральной ячейке $\left(\mathbf{R}_{i}=0\right.$, рис. 1) находится путем решения уравнения Шредингера

$$
\left[-\frac{\hbar^{2}}{2 m} \nabla^{2}+\delta v_{\mathrm{eff}, v}(r)-T_{0}\left(R_{N, v}\right)\right] u_{\mathrm{WS}}(r)=0
$$

с граничным условием

$$
\left.\nabla u_{\mathrm{WS}}(r)\right|_{r=R_{v}}=0
$$

т.е. $u_{\mathrm{WS}}(r)$ ведет себя как плоская волна на границе ячейки.

Решение уравнения (15) может быть упрощено путем замены потенциального профиля в ячейке на второе граничное условие

$$
\left.u_{\mathrm{WS}}(r)\right|_{r=L_{v}\left(R_{N, v}\right)}=0
$$

Для этого должно выполняться неравенство $L_{v}\left(R_{N, v}\right) / R_{v} \ll 1$. Величина $L_{v}\left(R_{N, v}\right)$ является длиной рассеяния электрона на вакансии в кластере.

Подстановка выражения для волновой функции электрона

$$
u_{\mathrm{WS}}(r)=\frac{A}{\sqrt{4 \pi}} \frac{\sin \left\{q_{0}\left[r-L_{v}\left(R_{N, v}\right)\right]\right\}}{q_{0} r}
$$

во второе граничное условие дает уравнение

$$
\operatorname{tg}\left[q_{0}\left(R_{v}-L_{v}\right)\right]-q_{0} R_{v}=0,
$$

из которого находится

$$
T_{0}=\frac{\hbar^{2} q_{0}^{2}}{2 m}
$$

Такой подход впервые использован в [18].

Размерная зависимость $T_{0}$ автоматически устанавливается по зависимости $L_{v}\left(R_{N, v}\right)=L_{v}+O\left(R_{N}^{-3}\right)[19]$. Вследствие этого можно пренебречь слабой зависимостью $T_{0}$ и $\left\langle\delta v_{\text {eff }, v}\right\rangle_{R_{v}}$ от $R_{N, v}$.

Здесь следует отметить, что расчеты по (20) и (14) согласуются друг с другом тем лучше, чем меньше $c_{v}$. Решение уравнения (19) в сверхячейке для $\mathrm{Al}$ при $c_{v}=0.01$ и 0.05 приводит к значениям $T_{0}=0.0388$ и $0.2535 \mathrm{eV}$ соответственно. Выражение (14) дает меньшие значения $\left(T_{0}=0.0296\right.$ и $\left.0.1480 \mathrm{eV}\right)$, что указывает на меньшую точность оптического приближения. 
Величина $\left\langle\delta v_{\mathrm{eff}, v}\right\rangle_{R_{v}}$ определяется выражением

$$
\begin{aligned}
\left\langle\delta v_{\mathrm{eff}, v}\right\rangle_{R_{v}} & =\int d \mathbf{r} \Psi^{*}(\mathbf{r}) \delta v_{\mathrm{eff}, v}(\mathbf{r}) \Psi(\mathbf{r}) \\
& =\frac{3 c_{v}}{4 \pi r_{0}^{3}} \int_{0}^{R_{v}} d r 4 \pi r^{2} \delta v_{\mathrm{eff}, v}(r),
\end{aligned}
$$

в которое удобно подставить $R_{v}=r_{0} c_{v}^{-1 / 3}$.

Качественно величину $\left\langle\delta v_{\mathrm{eff}, v}\right\rangle_{R_{v}}$ легко оценить, используя рис. $1, a$. Как нами было установлено (см. рис. 3 в [15]), для оценки можно принять $\delta v_{\text {eff, } v}(r) \approx 0$ уже при $r \approx 3 r_{0}$, несмотря на слабые фриделевские осцилляции; $r_{0}=3.99 a_{0}$ и $2.99 a_{0}$ для $\mathrm{Na}$ и $\mathrm{Al}$ соответственно. В большом кластере эффективный потенциал на границе кластера близок к потенциалу бесконечного образца и представляет собой яму глубиной $\bar{v}_{\text {eff }} \approx-6$ и $-15.5 \mathrm{eV}$ для $\mathrm{Na}$ и $\mathrm{Al}$ соответственно. Для грубой оценки потенциал вакансии может быть заменен потенциалом-бугром прямоугольной формы с высотой $U_{0}$ и радиусом $r_{0}$, отсчитываемым от $\bar{v}_{\text {eff }}$ - дна потенциала в объеме $\left(U_{0} \approx+2.5\right.$ и $+9 \mathrm{eV}$ для $\mathrm{Na}$ и $\mathrm{Al}$ соответственно $)$. Тогда

$$
\left\langle\delta v_{\mathrm{eff}, v}\right\rangle_{R_{v}} \approx U_{0} c_{v}
$$

На основе численного интегрирования с профилями потенциала, рассчитанного для 3D-металла с вакансией и без нее, можно рекомендовать более точную аппроксимацию

$$
\varepsilon^{(0)}=A_{1} c_{v}+A_{2} c_{v}^{2},
$$

где $A_{1}=4.10 \mathrm{eV}, A_{2}=3.70 \mathrm{eV}$ для $\mathrm{Na}$ и $A_{1}=13.3 \mathrm{eV}$, $A_{2}=28.25 \mathrm{eV}$ для $\mathrm{Al}$.

Т еория возмущений. Используя решение уравнения Шредингера в ячейке и выражение (13), можно записать уравнение для функции $\psi_{c}(\mathbf{r})$. Подставим (10) и (15) в уравнение (9), которое после простых преобразований может быть переписано в виде

$$
\begin{gathered}
{\left[-\frac{\hbar^{2}}{2 m} \nabla^{2}-\frac{\hbar^{2}}{m} \sum_{i=1}^{N_{v}} \frac{\nabla u_{\mathrm{WS}}\left(\left|\mathbf{r}-\mathbf{R}_{i}\right|\right)}{u_{\mathrm{WS}}\left(\left|\mathbf{r}-\mathbf{R}_{i}\right|\right)} \nabla+v_{\mathrm{eff}}(r)\right] \psi_{c}(\mathbf{r})} \\
=\varepsilon^{(1)} \psi_{c}(\mathbf{r}) .
\end{gathered}
$$

Уравнение (24) содержит „потенциальный профиль“ в виде перекрестного члена. Перекрестный член в (24) может быть учтен по теории возмущений. Ранее подобная процедура выполнялась с целью определения энергии основного состояния и эффективной массы электронов и позитронов в безграничных металлах. В [20,21] теория возмущений строилась на базисе ячеечных волновых функций, а $\psi_{c}(\mathbf{r})$ выбиралась в виде плоской волны. В [22] использовалось разложение $\psi_{c}(\mathbf{r})$ по плоским волнам в кристалле. В нашем случае внимание акцентировано на граничных условиях для $\psi_{c}(\mathbf{r})$ на поверхности, что приводит к дискретному энергетическому спектру частицы.
Определим энергию частицы в потенциальной яме $v_{\text {eff }}(r)$ всего кластера, полагая яму глубокой. Тогда граничное условие в уравнении (24) имеет вид

$$
\left.\psi_{c}(r)\right|_{r=R_{N, v}}=0
$$

После разложения $\psi_{c}(\mathbf{r})$ по базису волновых функций бесконечно глубокой ямы энергия $\varepsilon^{(1)}$ может быть представлена в удобной для дальнейших вычислений форме

$$
\varepsilon^{(1)}=\frac{\hbar^{2} \pi^{2}}{2 m R_{N, v}^{2}}+\langle\delta V(r)\rangle,
$$

при этом в качестве возмущения в (24) выбран оператор

$$
\delta V(r)=-\frac{\hbar^{2}}{m} \sum_{i=1}^{N_{v}} \frac{\nabla u_{\mathrm{WS}}\left(\left|\mathbf{r}-\mathbf{R}_{i}\right|\right)}{u_{\mathrm{WS}}\left(\left|\mathbf{r}-\mathbf{R}_{i}\right|\right)} \nabla
$$

Диагональный матричный элемент представляет собой поле $\delta V(r)$, усредненное по основному состоянию с квантовыми числами $n_{r}=1, l=0$,

$$
\langle\delta V(r)\rangle=\int_{r<R_{N, v}} d \mathbf{r} \psi(r) \delta V(r) \psi(r),
$$

где

$$
\psi(r)=\frac{1}{\sqrt{4 \pi}} \sqrt{\frac{2}{R_{N, v}}} \frac{\sin \left(\pi r / R_{N, v}\right)}{r} .
$$

Непосредственная оценка дает неожиданный результат

$$
\begin{aligned}
\langle\delta V(r)\rangle & \approx\left\langle\left(R_{v} \frac{\nabla u_{\mathrm{WS}}}{u_{\mathrm{WS}}}\right)\left(R_{N, v} \psi \nabla \psi\right)\right\rangle \\
& \approx \frac{\hbar^{2}}{m R_{v}^{2}} \frac{R_{v}}{R_{N, v}}
\end{aligned}
$$

где угловые скобки обозначают интегрирование по объему кластера.

Такая оценка, казалось бы, указывает на нарушение иерархии в разложении (26), так как на первый взгляд $\langle\delta V(r)\rangle$ имеет порядок $\left(R_{v} R_{N, v}\right)^{-1}$, в то время как предыдущий член имеет порядок $R_{N}^{-2}$. Однако интегрирование по углам в (28) вводит новый малый параметр

$$
\xi=\frac{L_{v}}{R_{v}}=\frac{L_{v}}{r_{0}} c_{v}^{1 / 3} \ll 1,
$$

и в результате появляется дополнительный множитель, пропорциональный произведению $\xi\left(\frac{R_{v}}{R_{N, v}}\right)$, т. е. другой малый параметр.

Вследствие того что возмущение $\delta V(r)$ происходит на масштабе ячейки, в (28) будет логичным перейти к интегрированию по ячейке и воспользоваться формулой 
Грина:

$$
\begin{aligned}
& \langle\delta V(r)\rangle=-\frac{\hbar^{2}}{2 m} \sum_{\substack{i=1 \\
L_{v}<\left|\mathbf{r}-\mathbf{R}_{i}\right|<R_{v}}}^{N_{v}} d \mathbf{r} \nabla\left\{\ln \left[u_{\mathrm{WS}}\left(\left|\mathbf{r}-\mathbf{R}_{i}\right|\right)\right]\right\} \nabla \psi^{2}(r) \\
& =-\frac{\hbar^{2}}{2 m} \sum_{i=1}^{N_{v}}\left[\left.\left\{\ln \left[u_{\mathrm{WS}}\left(\left|\mathbf{r}-\mathbf{R}_{i}\right|\right)\right]\right\}\right|_{\left|\mathbf{r}-\mathbf{R}_{i}\right|=R_{v}} \oint_{S_{i}} d \mathbf{S} \nabla \psi^{2}(r)\right. \\
& -\int_{L_{v}<\left|\mathbf{r}-\mathbf{R}_{i}\right|<R_{v}} d\left\{\left\{\ln \left[u_{\mathrm{WS}}\left(\left|\mathbf{r}-\mathbf{R}_{i}\right|\right)\right]\right\} \nabla^{2} \psi^{2}(r)\right] .
\end{aligned}
$$

Для дальнейших вычислений в (32) используем следующий прием.

Разложим функцию $\psi^{2}(r)$, медленно меняющуюся на масштабе ячейки, в градиентный ряд вблизи каждого значения $\mathbf{R}_{\mathbf{i}}$

$$
\begin{aligned}
\psi^{2}(r)= & \left.\psi^{2}(r)\right|_{r=R_{i}}+\left.\nabla \psi^{2}(r)\right|_{r=R_{i}}\left(\mathbf{r}-\mathbf{R}_{i}\right) \\
& +\left.\frac{\nabla^{2}}{2} \psi^{2}(r)\right|_{r=R_{i}}\left(r^{2}-2 \mathbf{r} \mathbf{R}_{i}+R_{i}^{2}\right)+\ldots
\end{aligned}
$$

Тогда для градиента и лапласиана от функции $\psi^{2}(r)$ при условии использования разложения $\psi^{2}(r)$ по степеням малости $\left|\mathbf{r}-\mathbf{R}_{i}\right| / R_{i} \equiv R_{v} / R_{i}$ имеем

$$
\begin{aligned}
\left.\nabla \psi^{2}(r)\right|_{R_{i} \gg R_{v}} & =\left[\frac{d \psi^{2}\left(R_{i}\right)}{d R_{i}}+\frac{d^{2} \psi^{2}\left(R_{i}\right)}{d R_{i}^{2}} \frac{R_{v}}{R_{i}}\right] \frac{\mathbf{r}}{r} \\
& =\frac{d \psi^{2}\left(R_{i}\right)}{d R_{i}}\left[1+O\left(\frac{R_{v}^{2}}{R_{i}^{2}}\right)\right] \frac{\mathbf{r}}{r}
\end{aligned}
$$

и в том же приближении

$$
\left.\nabla^{2} \psi^{2}(r)\right|_{R_{i} \gg R_{v}}=\left.\nabla^{2} \psi^{2}(r)\right|_{r=R_{i}}+O\left(\frac{R_{v}^{3}}{R_{i}^{3}}\right) .
$$

Подставляя эти выражения в (32), получим

$$
\begin{aligned}
\langle\delta V(r)\rangle= & -\frac{\hbar^{2}}{2 m} \sum_{i=1}^{N_{v}}\left[\left.\frac{d \psi^{2}\left(R_{i}\right)}{d R_{i}}\left(\ln u_{\mathrm{WS}}\right)\right|_{R_{v}}\right. \\
& \left.\times \oint_{S_{i}} d \mathbf{S} \frac{\mathbf{r}}{r}-\left.\nabla^{2} \psi^{2}(r)\right|_{R_{i}} \int_{i} d \mathbf{r} \ln u_{\mathrm{WS}}\right] .
\end{aligned}
$$

Используя выражение для волновой функции (18), получим

$$
\left.\left(\ln u_{\mathrm{WS}}\right)\right|_{r=R_{v}}=-\frac{3}{2}\left(\xi-\xi^{2}\right)+O\left(\xi^{3}\right)
$$

Очевидно, члены в правой части (36) являются конкурирующими.
Интеграл по поверхности $i$-й ячейки берется точно при произвольном значении $\mathbf{r}$ (рис. $1, b)$,

$$
\begin{aligned}
\oint_{S_{i}} d \mathbf{S} \frac{\mathbf{r}}{r} & =2 \pi R_{v}^{2} \int_{0}^{\pi} d \theta \cos \left(\theta_{i}-\theta\right) \sin \theta_{i} \\
& =2 \pi R_{v}^{2} \begin{cases}1-\frac{1}{3} \frac{R_{i}}{R_{v}}, & r<R_{v}, \\
\frac{2}{3} \frac{R_{v}}{R_{i}}, & r>R_{v},\end{cases}
\end{aligned}
$$

т.е. угловое интегрирование при $R_{i} \gg R_{v}$ дало дополнительную степень $R_{v} / R_{i}$. Переходя от суммирования к интегрированию в (36), с учетом множителя $R_{v} / R_{i}$ из (38) и выражения (29) имеем

$$
\begin{gathered}
\sum_{i=1}^{N} \frac{d \psi^{2}}{d R_{i}} \frac{1}{R_{i}}=\frac{3}{4 \pi R_{v}^{3}} \int_{0}^{R_{N}} d r 4 \pi r^{2} \frac{d \psi^{2}(r)}{d r} \frac{1}{r}=-\frac{3 C_{0}}{2 R_{v}^{3} R_{N, v}^{2}}, \\
C_{0}=-\int_{0}^{\pi} d y\left(\frac{\sin 2 y}{2 y}-\frac{\sin ^{2} y}{y^{2}}\right)=0.709
\end{gathered}
$$

Второй член в (36) после перехода к интегрированию по объему всего кластера может быть сведен к интегрированию по его поверхности. В этом случае вследствие граничного условия $\psi\left(R_{N, v}\right)=0$ он обращается в нуль. Окончательно для $\langle\delta V(r)\rangle$, учитывая (38) и (39), получим

$$
\langle\delta V(r)\rangle=-\frac{\hbar^{2} \pi^{2}}{2 m R_{N, v}^{2}}\left[\frac{12}{\pi} C_{0} \xi+O\left(\xi^{2}\right)\right]+O\left(\frac{R_{v}^{3}}{R_{N, v}^{3}}\right) .
$$

К числу отброшенных членов в квадратных скобках (41) относятся также вклады в энергию от „запрещенного“ объема в каждой ячейке, обусловленные наличием „твердых сердцевин“, другими словами, граничным условием $u_{\mathrm{WS}}\left(L_{v}\right)=0$ для ячеечной функции.

В результате, используя (6), (7), (23), (26) и (41), получаем окончательную формулу для потенциала ионизации, которая приобретает вид

$$
\begin{aligned}
\mathrm{IP}_{N, v}=W_{v}^{3 \mathrm{D}} & -\frac{\mu_{1}}{R_{N, v}}-\frac{\mu_{2}}{R_{N, v}^{2}} \\
& +\frac{e^{2}}{2 C_{N, v}}-\frac{\hbar^{2} \pi^{2}}{2 m R_{N, v}^{2}}\left(1-\frac{12}{\pi} C_{0} \xi\right),
\end{aligned}
$$

где параметр $\xi$ определяется (31).

Энергия прилипания ЕА выражается формулой (42), но с противоположным знаком энергии зарядки.

\section{4. Результаты и обсуждение}

Кластеры атомов обладают структурной периодичностью, которая скорее носит не трансляционный характер, а имеет свойство „сферической периодичности“ [23]. Эта периодичность обусловлена сферическими 
слоями атомов (атомными оболочками). Число атомов в кластерах с заполненными атомными слоями $N^{*}=13,55,147,309, \ldots$ Другая особенность, присущая только металлическим кластерам, обусловлена заполнением электронных оболочек по мере увеличения количества атомов. Кластеры с заполненными электронными оболочками обладают повышенной стабильностью по сравнению с кластерами других размеров, оболочки которых частично заполнены. Например, „магические“ числа для кластеров атомов $\mathrm{Na}$ и $\mathrm{Al}[24,25]$ $N^{* *}=8,20,40,58, \ldots$ и $13,22,35,66, \ldots$ соответственно. В квазиклассическом пределе разность между магическими и немагическими кластерами становится малозаметной.

По аналогии с отмеченным выше для кластеров с вакансиями мы можем ввести числа $N_{v}^{*}=N^{*}$, максимально соответствующие сферичности задачи. В расчете $\varepsilon^{(1)}$ было принято, что все сверхъячейки эквивалентны друг другу вплоть до поверхности кластера. В действительности поверхностные ячейки отличаются от объемных, т. е. частично „разрушены“. Оценим вклад пограничного слоя толщиной $x$ в диагональный матричный элемент $\varepsilon^{(1)}$. Для этого достаточно в (39) провести интегрирование от $\left(R_{N, v}-x\right)$ до $R_{N, v}$. В результате вклад от ячеек, размещенных в слое толщиной $x$ вблизи поверхности кластера, дает величину порядка $x /\left(R_{v} R_{N, v}\right)^{3}$, и поскольку $x \approx R_{v}$, сделанное предположение допустимо.

В связи с этим, а также с использованием условий сферической периодичности (10) и (16) минимальный размер кластера с вакансиями может быть выбран из условия $N_{v}^{*} \geq 13$. При $c_{v}=0.01$ для двух первых сферических оболочек $N_{v}^{*}=13,55$ полное число атомов в кластере $N=1300,5500$, что для $\mathrm{Al}$ соответствует $R_{N, v} \approx 1.8,2.9 \mathrm{~nm}$.

Однако более последовательным будет выбор границы применимости выражения (42) из условия разложения химического потенциала электронов нейтрального кластера в ряд по степеням обратного радиуса

$$
\mu_{1} R_{N} \geq \mu_{2}+\hbar^{2} \pi^{2} /(2 m) .
$$

В свою очередь это приводит к значениям $N \geq 9.4 \cdot 10^{3}(R \approx 4.5 \mathrm{~nm})$ и $N \geq 5.46 \cdot 10^{4}(R \approx 6 \mathrm{~nm})$ для $\mathrm{Na}$ и $\mathrm{Al}$ соответственно.

Большие размеры кластеров вполне детектируемы в эксперименте. Например, в работе [26] сообщалось о фотоэлектронной спектроскопии и фиксации ступеней кулоновской лестницы $\left(E_{C}=e^{2} / R_{N}\right)$ ионизованных кластеров $\mathrm{Al}_{N \leq 32000}^{-}$с точностью примерно $10^{-2} \mathrm{eV}$.

Для кластера с одной вакансией $\left(c_{v}=1 / N\right)$ выражение (10) неприменимо. Сверхъячейкой в определенном смысле является сам кластер. Условия на его границе приводят к уравнению $\sin \left[q_{0}\left(R_{N, v}-L_{v}\right)\right]=0$ для собственных значений волнового числа. В этом случае, удерживая первые поправки, получаем величину

$$
T_{0}=\frac{\hbar^{2} q_{0}^{2}}{2 m}=\frac{\pi^{2} \hbar^{2}}{2 m R_{N, v}^{2}}\left(1+\frac{2 L_{v}}{R_{N, v}}\right),
$$

причем интегрирование в (21) должно проводиться от 0 до $R_{N, v}$, а потенциал ионизации принимает вид

$$
\begin{gathered}
\mathrm{IP}_{N, v}=W_{0}-\left\langle\delta v_{\mathrm{eff}, v}\right\rangle_{R_{N, v}}-\frac{\pi^{2} \hbar^{2}}{2 m R_{N, v}^{2}}\left(1+\frac{2 L_{v}}{R_{N, v}}\right) \\
-\frac{\mu_{1}}{R_{N, v}}-\frac{\mu_{2}}{R_{N, v}^{2}}+\frac{e^{2}}{2 C_{N, v}}, \\
\left\langle\delta v_{\mathrm{eff}, v}\right\rangle_{R_{N, v}}=B c_{v}+D c_{v}^{2}, \quad c_{v}=1 / N
\end{gathered}
$$

где $B=2.72 \mathrm{eV}, \quad D=-2.0 \mathrm{eV} \quad$ и $\quad B=9.72 \mathrm{eV}$, $D=-1.50 \mathrm{eV}$ для $\mathrm{Na}$ и $\mathrm{Al}$ соответственно.

Выражения (42) и (44) для бездефектного кластера $\left(c_{v}=0\right)$ совпадают при $L_{v}=0$.

Величина $\delta_{1}$ в (4) определялась неоднократно [13]. Мы используем рассчитанные в модели стабильного желе [27] значения $\delta_{1}=\sigma_{1} / \sigma_{0}=0.32 r_{0}, 0.57 r_{0}$ для $\mathrm{Na}$ и $\mathrm{Al}$ соответственно $\left(\sigma_{1}=\gamma / 2\right.$, см. табл. VI работы [27]). Экспериментальные значения $W_{0}=2.75 \mathrm{eV}, \mathrm{IP}_{1}=5.14 \mathrm{eV}$ и $W_{0}=4.28 \mathrm{eV}, \mathrm{IP}_{1}=5.99 \mathrm{eV}$, а также $\sigma_{0} / B_{0}=0.70 a_{0}$ и $0.40 a_{0}$ для $\mathrm{Na}$ и $\mathrm{Al}$ соответственно взяты из [28].

Напомним дискуссию в литературе о коэффициентах $\alpha$ и $\beta$ первых размерных поправок потенциала ионизации и энергии прилипания [13]

$$
\begin{gathered}
\mathrm{IP}_{N}=W_{0}+\alpha \frac{e^{2}}{R_{N}}, \\
\mathrm{EA}_{N}=W_{0}-\beta \frac{e^{2}}{R_{N}},
\end{gathered}
$$

Величины $\alpha=3 / 8$ и $\beta=5 / 8$ были получены с использованием сил изображения. Затем было выяснено, что величины $\alpha=1 / 2-\mu_{1} / e^{2}$ и $\beta=1 / 2+\mu_{1} / e^{2}$ содержат параметр $\mu_{1}$, характерный для каждого материала, точно так же как и $W_{0}$.

Формулы (46) являются результатом непрерывной модели в квазиклассическом приближении, когда флуктуации числа атомов на единицу в кластере и дискретность спектра электронов не влияют на положение химического потенциала электронов. Использование формул (46) для кластеров, состоящих уже из сотен атомов, является обычной экстраполяцией. Следует иметь в виду условия эксперимента, при которых получаются значения IP и ЕА. В случае масс-спектрометрических измерений, в которых значения IP и ЕА однозначно сопоставляются с числом атомов $N$ в кластере, формула (46) является опорной для анализа размерной зависимости $\mathrm{IP}_{N}$ (см., например, недавние измерения для $\mathrm{Al}_{N=32-95}$ в [11]).

На рис. 2 для $\mathrm{Na}$ приведены зависимости (46) и (42). Вследствие того что формула (46) дает значение $\mathrm{IP}_{1}$ всего на 10\% выше экспериментального, предполагалось по измерениям $\mathrm{IP}_{N \leq 100}$ для магических кластеров (см. рис. 28 в [24]) с применением (46) определять истинные значения $W_{0}$. Экспериментальные значения $W_{0}$ для ряда материалов сильно зависят от методик измерения. С другой стороны, размерные поправки $\sim R_{N, v}^{-2}$, а именно последнее слагаемое в (42), обусловленное квантованием, приводят к патологическому поведению 


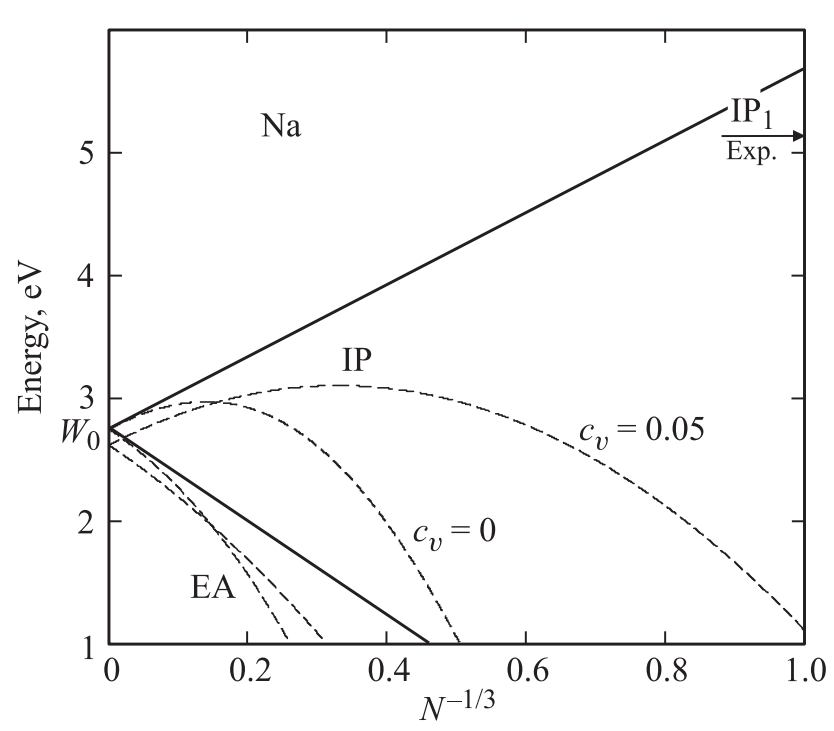

Рис. 2. Расчетные зависимости $\mathrm{IP}_{N, v}$ и $\mathrm{EA}_{N, v}$ для $\mathrm{Na}$ в приближениях (46) (сплошные линии) и (42) для $c_{v}=0$ и 0.05 (штриховые линии).

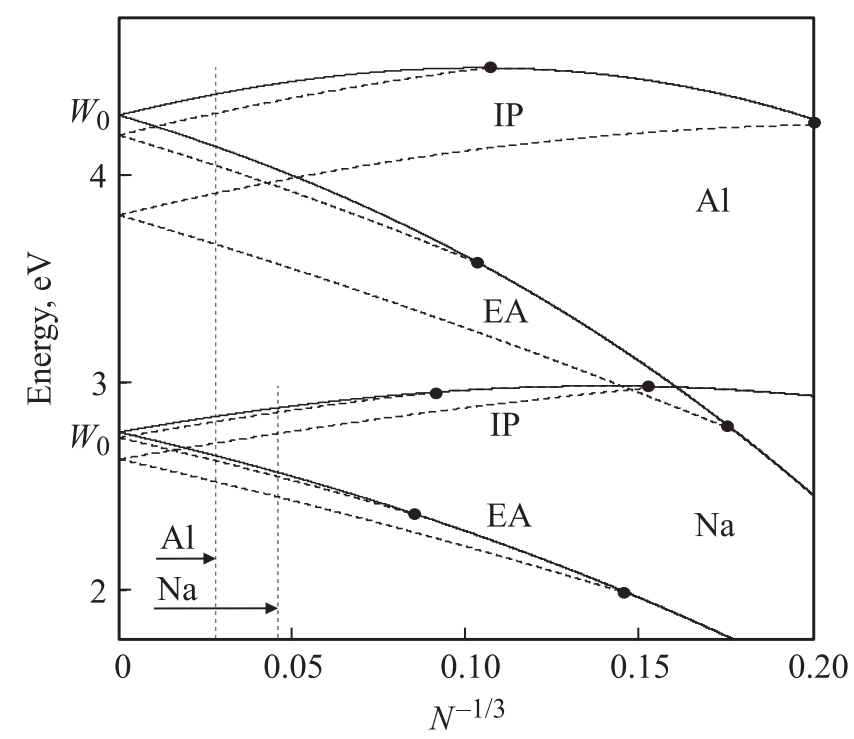

Рис. 3. Зависимости $\mathrm{IP}_{N, v}$ и $\mathrm{EA}_{N, v}$ для больших кластеров $\mathrm{Na}$ и $\mathrm{Al}$, рассчитанные по формулам (44) (сплошные линии, $\left.c_{v}=1 / N\right)$ и (42) для $c_{v}=0.01$ (верхние штриховые линии) и 0.05 (нижние штриховые линии).

$\mathrm{IP}_{N}$ и $\mathrm{EA}_{N}$ в области малых $N$. Такое поведение демонстрируют на рис. 2 зависимости (42), построенные для значений концентрации вакансий $c_{v}=0$ и 0.05 , что заставляет нас акцентировать внимание на границе применимости выражения (43) и более детально исследовать эти зависимости вблизи начала координат.

Рис. 3 позволяет судить о точности модели и определить ее адекватность при различных условиях или возможных измерениях. На рис. 3 приведены зависимости для больших кластеров $\mathrm{Na}$ и $\mathrm{Al}$, рассчитанные по формуле (44) с $c_{v}=1 / N$ (сплошные линии) и форму- ле (42) для значений $c_{v}=0.01,0.05$ (штриховые линии). Сплошные линии являются опорными $\left(c_{v} \geq 1 / N\right)$, а точки пересечения штриховых линий со сплошными обозначены темными кружками. По мере роста концентрации вакансий увеличивается щель $\varepsilon^{(0)}$. Именно на эту величину сдвигается значение работы выхода $W_{0}$ на оси ординат. Вертикальными линиями помечены границы применимости данного разложения. Можно отметить заметную вакансионную зависимость кривых как для потенциала ионизации, так и для энергии прилипания.

Предложенный подход представляется перспективным для экспериментального определения концентраций точечных дефектов или примесей в кластерах металлов. Для этого предварительно нужно рассчитать длину рассеяния электронов на соответствующем дефекте в 3D-металле. В частности, может быть решен вопрос о концентрации вакансий в кластере при температуре плавления. Если использовать рис. 3 как сетку значений, при попадании экспериментального значения $\mathrm{IP}_{N, v}$ на одну из кривых фиксируется величина $c_{v}(N)$ при заданной температуре.

Общим недостатком моделей типа модели желе является допущение о сферической форме кластеров атомов металла.

\section{5. Заключение}

В работе предложена последовательная процедура нахождения потенциала ионизации большого металлического кластера, содержащего вакансии, которая базируется на предварительно решенной задаче о рассеянии электронов на моновакансии в массивном металле методом Кона-Шема в модели стабильного желе. Самосогласованные профили потенциалов используются для определения вакансионного сдвига энергии основного состояния в металлическом кластере-сфере в виде серии размерных поправок. Предполагалась сферическая периодичность в расположении вакансий. Определены границы применимости данного разложения по степеням обратного радиуса $R^{-1}: R>4.5 \mathrm{~nm}$ и $R>6 \mathrm{~nm}$ для $\mathrm{Na}$ и $\mathrm{Al}$ соответственно.

Данный подход представляется перспективным для экспериментального определения концентраций точечных дефектов или примесей в кластерах металлов. Для этого предварительно нужно рассчитать длину рассеяния электронов на соответствующем дефекте в 3Dметалле. Полученные аналитические выражения удобны для анализа результатов фотоионизационных экспериментов. С их помощью, в частности, может быть определена концентрация вакансий в кластере вблизи температуры плавления. При попадании экспериментального значения потенциала ионизации IP (или энергии прилипания EA) на одну из рассчитанных размерных и концентрационных зависимостей IP (или ЕА) автоматически фиксируется размер кластера и концентрация вакансий при заданной температуре. 


\section{Список литературы}

[1] R.S. Berry, B.M. Smirnov. ЖЭТФ 125, 414 (2004).

[2] R.S. Berry, B.M. Smirnov. Phys. Rep. 527, 205 (2013).

[3] А.В. Бабич, В.В. Погосов, В.И. Рева. ФТТ. 57, 2081 (2015).

[4] C. Hock, C. Bartels, S. Straßburg, M. Schmidt, H. Haberland, B. von Issendorff, A. Aguado. Phys. Rev. Lett. 102, 043401 (2009).

[5] C.C. Yang, S. Li. Phys. Rev. B 75, 165413 (2007).

[6] G. Guisbiers. Nanoscale Res. Lett. 5, 1132 (2010).

[7] G.A. Breaux, C.M. Neal, B. Cao, M.F. Jarrold. Phys. Rev. Lett. 94, 173401 (2005).

[8] A.K. Starace, B. Cao, O.H. Judd, I. Bhattacharyya, M.F. Jarrold. J. Chem. Phys. 132, 034302 (2010).

[9] C. Bréchignac, Ph. Cahuzac, J. Leygnier, J. Weiner. J. Chem. Phys. 90, 1492 (1989).

[10] U.R. Martin, F. Jarrold, J.E. Bower, J.S. Kraus. J. Chem. Phys. 91, 2912 (1989).

[11] A. Halder, V.V. Kresin. J. Chem. Phys. 143, 164313 (2015).

[12] J.P. Perdew, M. Brajczewska, C. Fiolhais. J. Chem. Phys. 108, 8182 (1998).

[13] В.В. Погосов. Введение в физику зарядовых и размерных эффектов: поверхность, кластеры, низкоразмерные системы. Физматлит, М. (2006). 328 с.

[14] А.В. Бабич, П.В. Вакула, В.В. Погосов. ФТТ. 56, 841 (2014).

[15] А.В. Бабич, П.В. Вакула, В.В. Погосов. ФТТ. 56, 1671 (2014).

[16] V.V. Pogosov, W.V. Pogosov, D.P. Kotlyarov. ЖЭТФ. 117, 1043 (2000).

[17] В.В. Погосов. ФТТ 35, 1010 (1993).

[18] B.E. Springett, M.H. Cohen, J. Jortner. Phys. Rev. 159, 183 (1967).

[19] I.T. Iakubov, V.V. Pogosov. J. Chem. Phys. 106, 2306 (1997).

[20] J. Bardeen. J. Chem. Phys. 6, 367 (1938).

[21] M.H. Cohen, F.S. Ham. J. Phys. Chem. Sol. 16, 177 (1960).

[22] M.J. Stott, P. Kubica. Phys. Rev. B 11, 1 (1975).

[23] T.P. Martin. Phys. Rep. 273, 199 (1996).

[24] W.A. de Heer. Rev. Mod. Phys. 65, 611 (1993).

[25] M. Brack. Rev. Mod. Phys. 65, 677 (1993).

[26] M.A. Hoffmann, G. Wrigge, B. von Issendorff. Phys. Rev. B 66, 014404 (2002).

[27] P. Ziesche, J.P. Perdew, C. Fiolhais. Phys. Rev. B 49, 7919 (1994).

[28] J.A. Alonso, N.M. March. Surf. Sci. 160, 509 (1985). 